\title{
BMJ Open Effectiveness of interventions targeting antibiotic use in long-term aged care facilities: a systematic review and meta- analysis
}

\author{
Magdalena Z Raban (D) , ${ }^{1}$ Claudia Gasparini, ${ }^{1}$ Ling Li, ${ }^{1}$ Melissa T Baysari (D) , ${ }^{1,2}$ \\ Johanna I Westbrook ${ }^{1}$
}

To cite: Raban MZ, Gasparini C, Li L, et al. Effectiveness of interventions targeting antibiotic use in long-term aged care facilities: a systematic review and meta-analysis. BMJ Open 2020;10:e028494. doi:10.1136/ bmjopen-2018-028494

- Prepublication history and additional material for this paper are available online. To view these files, please visit the journal online (http://dx.doi. org/10.1136/bmjopen-2018028494).

Received 11 December 2018 Revised 05 December 2019 Accepted 18 December 2019

A) Check for updates

(c) Author(s) (or their employer(s)) 2020. Re-use permitted under CC BY-NC. No commercial re-use. See rights and permissions. Published by BMJ.

${ }^{1}$ Faculty of Medicine and Health Sciences, Australian Institute of Health Innovation, Macquarie University, Sydney, New South Wales, Australia

${ }^{2}$ Faculty of Health Sciences, The University of Sydney, Sydney, New South Wales, Australia

Correspondence to Dr Magdalena Z Raban; magda.raban@mq.edu.au

\section{ABSTRACT}

Objectives There are high levels of inappropriate antibiotic use in long-term care facilities (LTCFs). Our objective was to examine evidence of the effectiveness of interventions designed to reduce antibiotic use and/or inappropriate use in LTCFs.

Design Systematic review and meta-analysis.

Data sources MEDLINE, Embase and CINAHL from 1997 until November 2018.

Eligibility criteria Controlled and uncontrolled studies in LTCFs measuring intervention effects on rates of overall antibiotic use and/or appropriateness of use were included. Secondary outcomes were intervention implementation barriers from process evaluations. Data extraction and synthesis Two reviewers independently applied the Cochrane Effective Practice and Organisation of Care group's resources to classify interventions and assess risk of bias. Meta-analyses used random effects models to pool results.

Results Of include studies ( $n=19), 10$ had a control group and 17 had a high risk of bias. All interventions had multiple components. Eight studies (with high risk of bias) showed positive impacts on outcomes and included one of the following interventions: audit and feedback, introduction of care pathways or an infectious disease team. Meta-analyses on change in the percentage of residents on antibiotics (pooled relative risk (RR) (three studies, 6862 residents): $0.85,95 \% \mathrm{Cl}: 0.61$ to 1.18 ), appropriateness of decision to treat with antibiotics (pooled RR (three studies, 993 antibiotic orders): 1.10, $95 \%$ Cl: 0.64 to 1.91 ) and appropriateness of antibiotic selection for respiratory tract infections (pooled RR (three studies, 292 orders): $1.15,95 \% \mathrm{Cl}: 0.95$ to 1.40), showed no significant intervention effects. However, meta-analyses only included results from intervention groups since most studies lacked a control group. Insufficient data prevented meta-analysis on other outcomes. Process evaluations $(n=7)$ noted poor intervention adoption, low physician engagement and high staff turnover as barriers.

Conclusions There is insufficient evidence that interventions employed to date are effective at improving antibiotic use in LTCFs. Future studies should use rigorous study designs and tailor intervention implementation to the setting.
Strengths and limitations of this study

We used a broad search strategy to identify studies evaluating the impact of interventions aiming to improve antibiotic use in long-term care facilities and considered a range of detailed indicators of antibiotic use and appropriateness.

- To support comparison with future reviews, intervention components were classified using the Cochrane Effective Practice and Organisation of Care group's Taxonomy of Health System Interventions.

- We report the results of process evaluations to identify reasons for intervention effects, in order to inform future intervention design and implementation.

- A limitation of the meta-analyses is that these were conducted using pre-intervention and postintervention measurements from the intervention groups of studies, as there were insufficient studies with control groups to include in the analyses.

- The majority of included studies were assessed as having a high risk of bias.

\section{INTRODUCTION}

The widespread inappropriate use of antibiotics is a major contributor to antibiotic resistance. Antibiotic resistance is a growing problem globally, leading to poor patient outcomes, pressure on health system resources and significant economic impacts. ${ }^{1-6}$ Antimicrobial resistant infections are estimated to cause over 700000 deaths annually worldwide- - a rate which is projected to increase to 10 million by $2050 .^{1}$

In long-term care facilities (LTCFs), antibiotic use rates are high compared with the general population, and LTCFs residents are on antibiotics for longer. ${ }^{7}$ Studies have estimated that between $47 \%$ and $70 \%$ of residents receive a course of antibiotics annually, and that between $77 \%$ to $88 \%$ of infections are treated with an antibiotic. ${ }^{7-9}$ Approximately $50 \%$ of these prescriptions are judged to be inappropriate, ${ }^{9}$ with inappropriate 
treatment of urinary tract infections and respiratory tract infections common in LTCF residents. ${ }^{7-12}$ Thus, there is considerable scope for improving antibiotic use in LTCFs. This goal is especially important given that residents of LTCFs are at increased risk of colonisation with resistant organisms and facilitate the spread of these organisms due to their close proximity to other residents and regular contact with hospitals, LTCF workers and the community. ${ }^{13-18}$ The widespread use of antibiotics by LTCF residents also puts them at risk of related infections, such as Clostridioides difficile, which are subsequently easily spread to contacts. ${ }^{19}$

Interventions designed to improve antibiotic prescribing in the hospital setting have been shown to confer benefits in terms of appropriate antibiotic use, such as adherence to antibiotic use policy and shorter antibiotic course length. ${ }^{20}$ Similarly, interventions targeting physicians in primary care have been shown to reduce rates of antibiotic prescribing and improve compliance with guidelines. ${ }^{21}{ }^{22}$ Evidence of effective interventions in LTCFs is more limited, and the LTCF setting is complex with many factors that can influence the success of interventions. Two previous systematic reviews of interventions to improve antibiotic use in LTCFs both concluded that while there was some evidence to suggest these interventions had positive impacts, the evidence base needed strengthening. ${ }^{23} 24$ However, there is an opportunity to build on this work. One of these reviews, published in 2013, included only randomised trials of which there were only four. ${ }^{24}$ The second review's primary aim was to assess whether antimicrobial stewardship interventions in LTCFs reduced mortality, the incidence of Clostridioides difficile infections, emergency department visits and hospitalisations. ${ }^{23}$ The impact of interventions on antibiotic use was a secondary objective. Neither review included a metaanalysis of the effectiveness of interventions to reduce and improve antibiotic use, nor examined factors influencing intervention effectiveness.

Our objective was to conduct a systematic review and meta-analysis of the effectiveness of interventions to reduce the use or improve the appropriateness of antibiotics in LTCFs. The primary outcomes of interest were changes in rates of overall antibiotic use and rates of appropriate antibiotic use. In order to gain insights into how intervention impacts were achieved, the secondary aim was to provide a qualitative review of the results from process evaluations that were conducted as part of the intervention studies.

\section{METHODS}

This systematic review and meta-analysis are reported according to the Preferred Reporting Items for Systematic Reviews and Meta-Analyses ${ }^{25}$ (online supplementary file 1).

\section{Information sources and search strategy}

The databases MEDLINE via Ovid (1946 to February 2018) and via PubMed (1966 to February 2018), Embase (1974 to February 2018) and CINAHL (1966 to February 2018) were searched in November 2018 for original research articles reporting on the impact of interventions to improve antibiotic use in LTCFs, published in English, in the last 20 years (since 1997). The search used keywords and subject heading terms related to two main groups of concepts which were combined using the Boolean operator 'AND': (i) long-term care facilities and (ii) antibiotics, common infections affecting long-term care residents and antibiotic resistant organisms. The full search strategy is presented in online supplementary file 2. The reference lists of included articles and identified review articles were hand searched for further relevant citations. Additionally, the PROSPERO: International Prospective Register of Systematic Reviews ${ }^{26}$ and the Cochrane Library ${ }^{27}$ were searched.

\section{Eligibility criteria}

Original research articles reporting the effects of interventions to reduce the use of antibiotics or improve the appropriateness of antibiotic use in residents of LTCFs were eligible for inclusion. Studies in residential aged care facilities, nursing homes, skilled nursing facilities and assisted living facilities for older people were included; while those in community-dwelling populations, and long-term care hospital wards were excluded. Long-term care hospital wards have a different model of care and populations compared with stand-alone LTCFs. Studies were included if they reported intervention outcomes in terms of the rate of overall antibiotic use or rate of appropriate antibiotic use (eg, per resident, per 1000 bed days, per antibiotic prescription). Studies reporting only rates of infection, resistant organism colonisation, hospitalisation or mortality were excluded, as were studies where the intervention did not target antibiotic use for example, interventions consisting only of infection control strategies. Due to the limited literature on this topic, the study designs were not restricted to randomised trials. The study types eligible for inclusion were: randomised trials, controlled before and after studies, interrupted time series and before and after studies. Crosssectional studies were not eligible for inclusion.

\section{Study selection}

Two authors (MR, CG) independently reviewed the title and abstracts of citations returned from the search strategy, after removal of duplicates, to identify potentially relevant citations for full-text review. The full-text of all relevant citations were then reviewed independently by two authors (MR, CG) using Covidence software to aid the process. ${ }^{28}$ Discrepancies between the two authors in the assessment of full-text articles were resolved through discussion.

\section{Data collection and data items}

Data were extracted initially by one author (CG), and then this was reviewed and refined by a second author 
(MR). The data extracted included study characteristics (country, number of LTCFs included, study design), outcomes of interest measured and results related to these and intervention details. For overall antibiotic use outcomes, the indicators extracted were the standard indicators used to report antibiotic use: $:^{29-31}$ defined daily doses per 1000 resident days (DDD/1000 days), days of antibiotic therapy per 1000 resident days (DOT/1000 days), prescriptions (equivalent to antibiotic initiations) per 1000 resident days, and percentage of residents on an antibiotic. Indicators of the appropriateness of antibiotic use extracted were: the percentage of antibiotic prescriptions that were appropriate decisions to treat an infection with an antibiotic (appropriateness of decisions to treat), and the percentage of antibiotic prescriptions where the appropriate antibiotic was selected (appropriateness of antibiotic selection). The methods for assessment of antibiotic appropriateness were recorded, and the appropriateness outcomes were stratified by infection type (all infections, urinary tract infections (UTIs), respiratory tract infections (RTIs)). The most common infection types in LTCF are UTIs, RTIs and skin and soft tissue infections, accounting for over $90 \%$ of all infections. ${ }^{732} 33$ If interventions targeted all three infection types, they were classified as targeting 'all infections'. The timing of outcome measurement in relation to the beginning of intervention implementation (excluding any piloting of the intervention) was also recorded. When studies included process evaluations, the results of the process evaluation were extracted.

Intervention details extracted included the infection types and personnel who were targeted by the intervention (eg, nurses, physicians, family/residents). The Cochrane Effective Practice and Organisation of Care (EPOC) group's Taxonomy of Health System Interventions was used as a guide to classify intervention components. ${ }^{34}$ Two authors (CG and MR) independently coded the study interventions and resolved discrepancies in coding by discussion. Online supplementary file 3 shows the intervention taxonomy sub-categories applied, along with their definitions.

\section{Assessment of risk of bias in included studies}

The risk of bias was assessed using the Cochrane EPOC group's criteria. ${ }^{35}$ An assessment of the risk of bias of studies provides an indication of the likely issues that may bias study results. The Cochrane EPOC group's risk of bias tool is an extension of the Cochrane Collaboration's tool and includes items specific not only to randomised trials, but non-randomised trials and controlled beforeand-after studies. The included studies were rated against each criterion as having either a low risk, high risk or unclear risk of bias.

The criterion assessing whether outcome assessment was blinded was applied to each of the two categories of outcomes of interest (overall antibiotic use and appropriate antibiotic use). Within this criterion we also considered whether the study assessed the reliability of outcome measurement. This is especially important when considering the appropriateness of antibiotic use as it requires clinical judgement, and previous studies have shown that this can vary between assessors. ${ }^{36-38}$ Studies without controls (interrupted time series or before-after designs) were rated against three additional criteria.

We considered whether the characteristics of the facility residents were similar in the before and after periods. Differences in the characteristics of the residents in the two periods could affect the outcomes of interest. We also added two additional criteria related to long follow-up periods (where other systemic changes may influence outcomes) and retrospective study designs, which increase the risk of bias of before-after study designs. ${ }^{39}$ Criteria requiring assessment of the comparability of the control and intervention groups were not considered as they are not applicable in these study designs.

The risk of bias for all studies was rated independently by two authors (MR and MB). Discrepancies in assessment were resolved through discussion to arrive at a final rating for each criterion. The risk of bias assessment tools used are presented in full in online supplementary file 4 . A summary of risk of bias assessment score was assigned to each study as follows: 'low risk of bias' when all criteria were scored as 'low', 'medium risk of bias' if one to two criteria were scored as 'unclear' or 'high', and 'high risk of bias' if more than two criteria were scored as 'unclear' or 'high'. RevMan ${ }^{40}$ software was used to collate and present the risk of bias results.

\section{Meta-analysis}

Authors of papers containing insufficient information to be included in the meta-analyses were sent emails requesting additional data to be provided. Where sufficient data were available, we conducted meta-analyses for the outcomes of interest using random effects models to pool the results. Since less than three studies for any given outcome included control groups, only the results from the intervention groups were included in the metaanalyses. Relative risk ratios (RR) and their 95\% CIs were calculated. The conservative Knapp-Hartung approach ${ }^{41}$ was applied to account for heterogeneity between studies. The meta-analyses results are presented using forest plots. Between-study heterogeneity was evaluated using $\mathrm{I}^{2}$ statistics. ${ }^{42}$ The potential for publication bias for each metaanalysis was assessed by inspection of funnel plots and statistical tests based on weighted linear regression of the intervention effect on its SE. ${ }^{43}$ Sensitivity analyses were conducted using ORs. In addition, analyses were also conducted for studies reporting results from different post-intervention periods ${ }^{44} 45$ and different levels of appropriateness of antibiotic selection affected results. ${ }^{46}$ All statistical tests were two-sided and were evaluated at a significance level of 0.05 . Analyses were carried out using R V.3.5.0. ${ }^{47}$ 


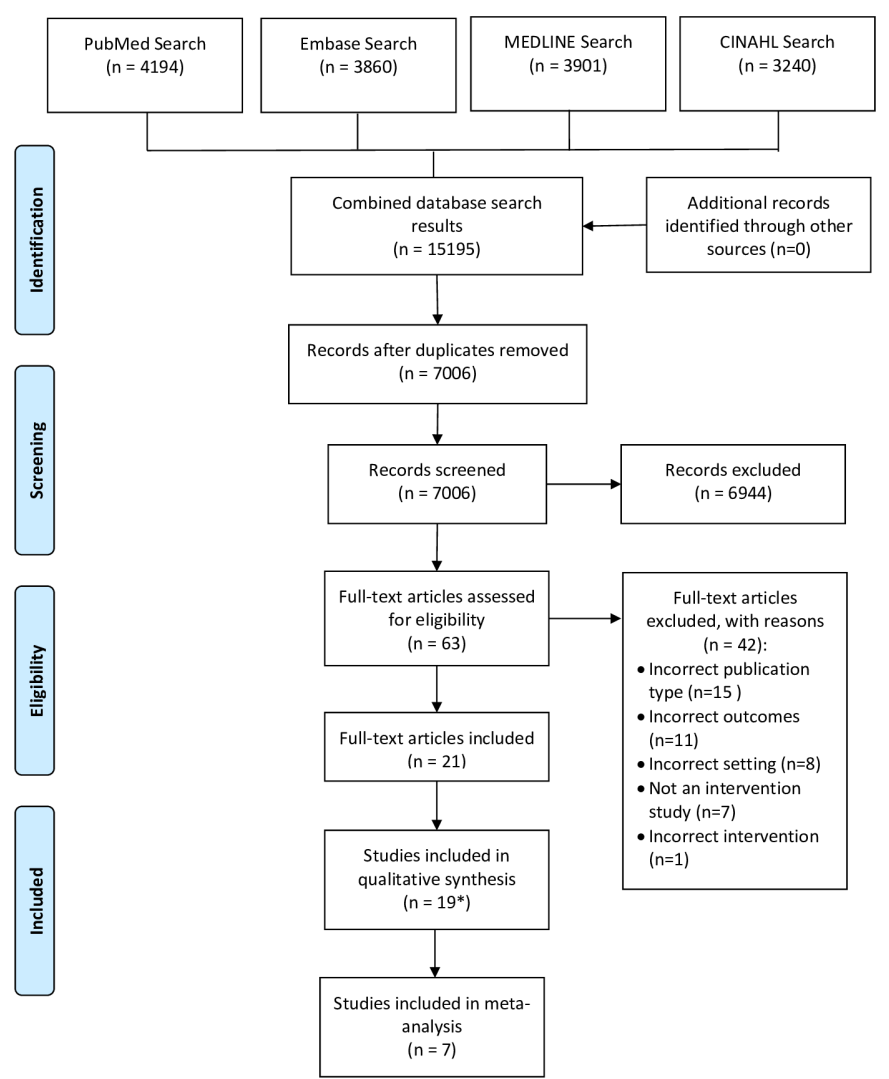

Figure 1 Preferred Reporting Items for Systematic Reviews and Meta-Analyses flowchart of search strategy and screening results. *There were two instances where two fulltext articles reported results from one study.

\section{Patient and public involvement}

There was no patient or public involvement in the design or conduct of this study.

\section{RESULTS}

There were 19 studies (21 papers) assessed as eligible for inclusion in this review (figure 1). ${ }^{44-46}$ 48-63 Table 1 presents the study characteristics. The majority of studies were undertaken in North America $(n=14)$, four in Europe and one in Australia. Five were cluster randomised trials, ${ }^{465457-5961}$ five were controlled beforeafter studies, ${ }^{50} 53 \quad 56 \quad 62 \quad 64$ two were interrupted time series studies ${ }^{49} 51$ and there were seven before-after studies. 46485260616365 Thirteen studies measured overall antibiotic use and 10 the appropriateness of antibiotic use. Data were received in 3 out of 10 cases. Information reported in included studies or reported by authors allowed us to perform meta-analyses for three outcome measures: percentage of residents on antibiotics, appropriateness of decision to treat (any infection) and appropriateness of antibiotic selection (for RTIs).

\section{Description of interventions}

Interventions were aimed at improving antibiotic use for all infection types in eight studies, 4546515759616465 UTIs in five studies, ${ }^{4952545663}$ RTIs in three studies ${ }^{5053} 58$ and both UTIs and RTIs in three studies ${ }^{48} 6062$ (table 1). The health professionals targeted by interventions were most commonly both physicians and nurses $(n=14)$. Four studies ${ }^{5260164}$ also targeted facility residents and/ or their families (table 1). All studies used interventions involving multiple components (table 2). Education strategies were an intervention component in the majority of studies $(n=17),{ }^{46} 4850-5456-64$ followed by the promotion of clinical practice guidelines $(\mathrm{n}=15),{ }^{45} 46$ 48-50 52-54 56-59 6264 which in a further seven studies were adapted to the local context. $^{45} 485356-5860$ Audit and feedback interventions used in five studies involved reporting on antibiotic prescribing to the facility or to physicians. ${ }^{52} 5763-65$ Four studies assessed an intervention which included an infectious disease team who provided advice to physicians on appropriate antibiotic use for individual cases of infection. ${ }^{49} 516065$ The least common intervention component was information and communication technology (ICT; $\mathrm{n}=2),{ }^{48}$ which was used in one study to provide physicians with prescribing guidelines, ${ }^{48}$ and in the other study to change default stop dates for some antibiotics, as well as improved ease of access to guidelines. ${ }^{52}$

\section{Risk of bias in included studies}

All but two studies ${ }^{54}{ }^{57}$ were rated as having an overall high risk of bias (table 1). Figure 2 shows the risk of bias assessments against each of the criteria for each study. In studies with controls, allocation concealment and random sequence generation were identified to be at a high risk of bias in eight and five studies, respectively. This is largely due to the high proportion of controlled beforeafter studies which did not undertake a randomisation process. ${ }^{35}$ The blinding and reliability of measurement of antibiotic appropriateness outcomes were judged to be at a high risk of bias in all studies with controls $(n=7)$.

Eight of nine studies without controls did not consider the potential bias of changes in resident characteristics in the before-and-after intervention periods, and thus had a high risk of bias for this criterion (figure 2). Similarly, to controlled studies, the two studies without controls reporting appropriateness of antibiotic use outcomes had a high risk of bias for the blinding and reliability criterion.

Online supplementary file 5 presents the summary of the risk of bias assessment.

\section{Overview of intervention effectiveness}

Of the included studies, only eight showed a statistically significant improvement in the outcomes of interest (table 2). ${ }^{45} 4649-51$ 63-65 These studies used three intervention components more frequently compared with the studies without significant effects on outcomes. These components included: audit and feedback (three ${ }^{63-65}$ of five $^{525763-65}$ studies had significant effects), introduction of care pathways (three ${ }^{465064}$ of five $\mathrm{e}^{465536164}$ studies had significant effects) and use of an infectious disease team (three ${ }^{495165}$ of four ${ }^{45515765}$ studies had significant effects). However, five ${ }^{4549516365}$ of the eight studies that showed an improvement in outcomes used study designs 


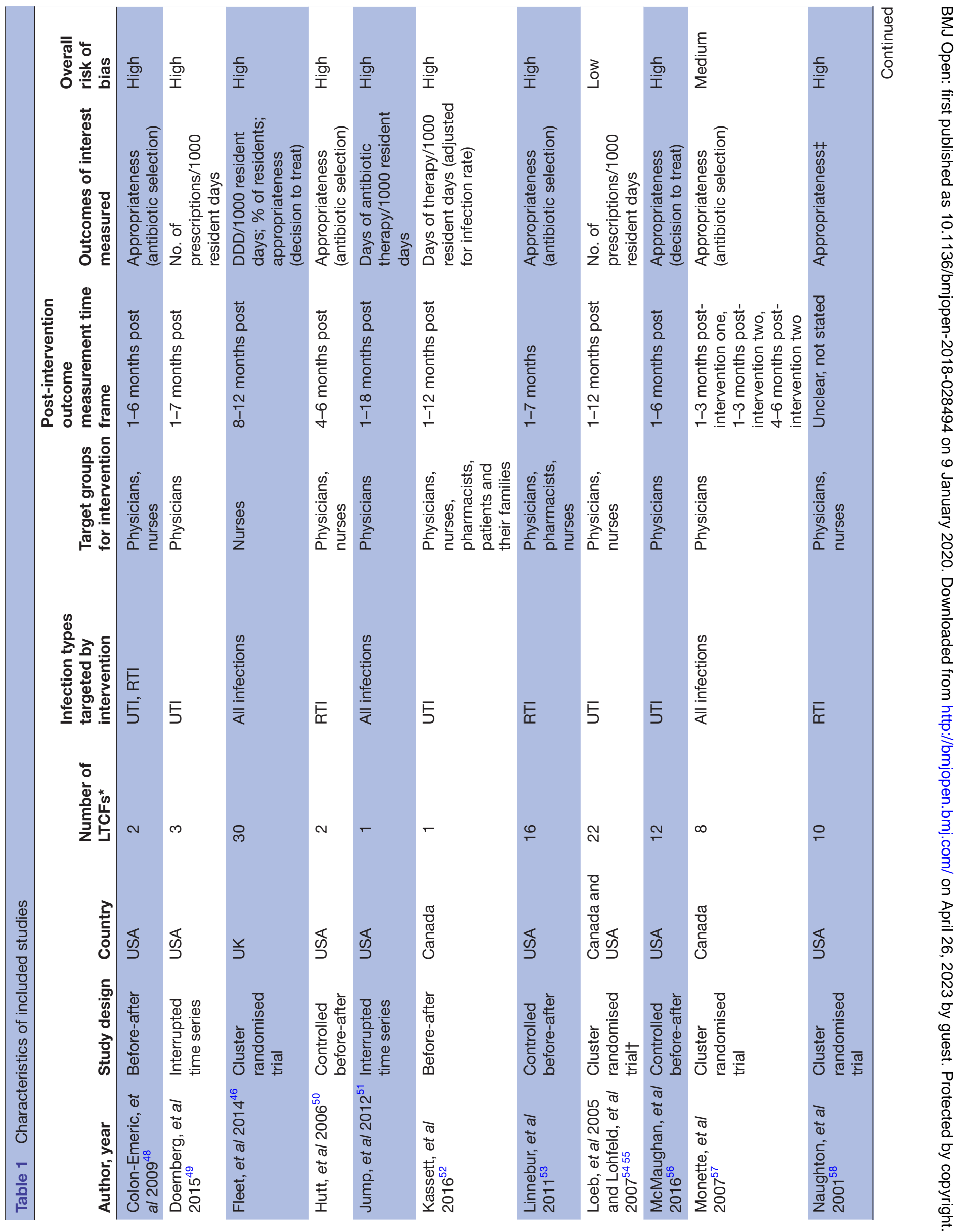




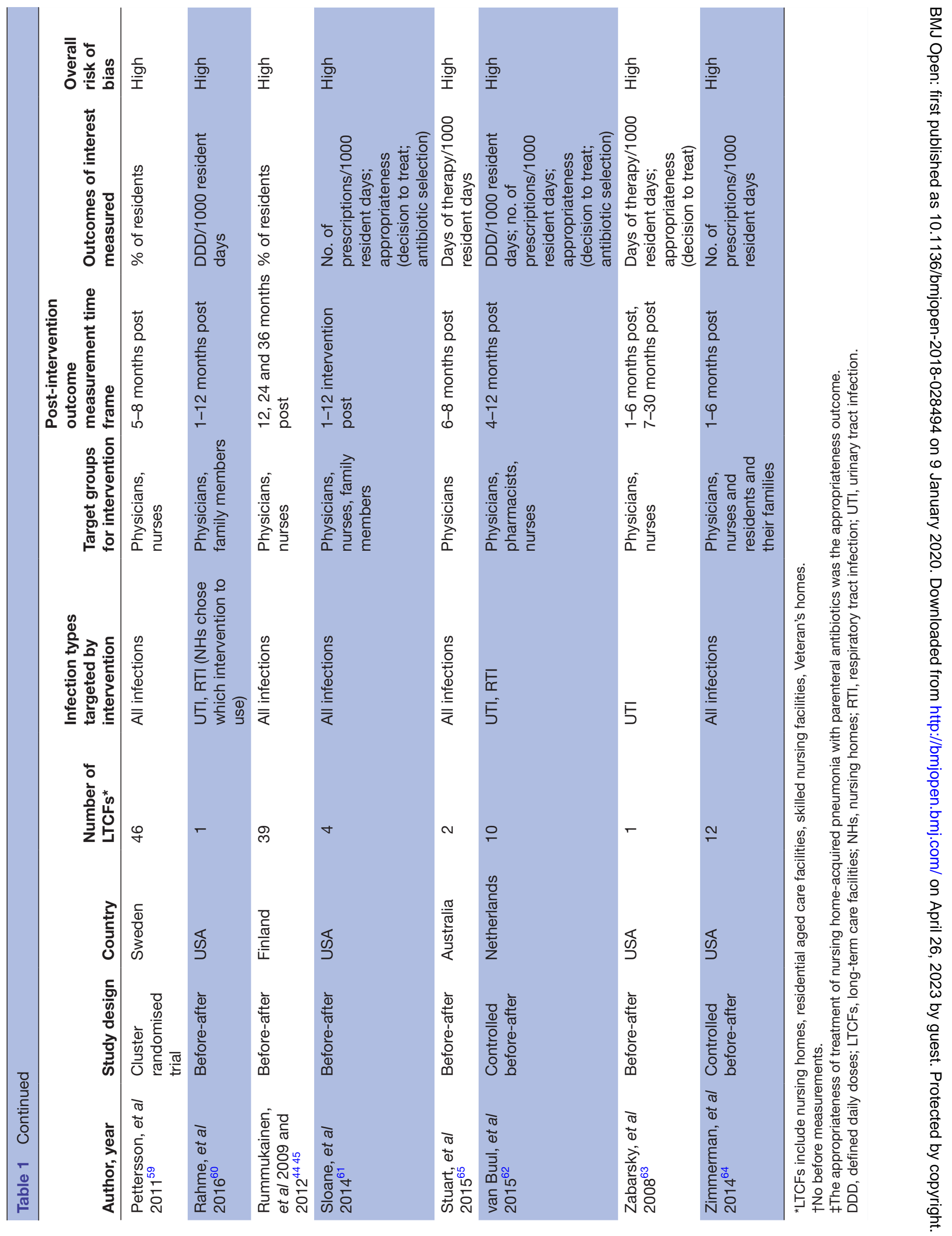




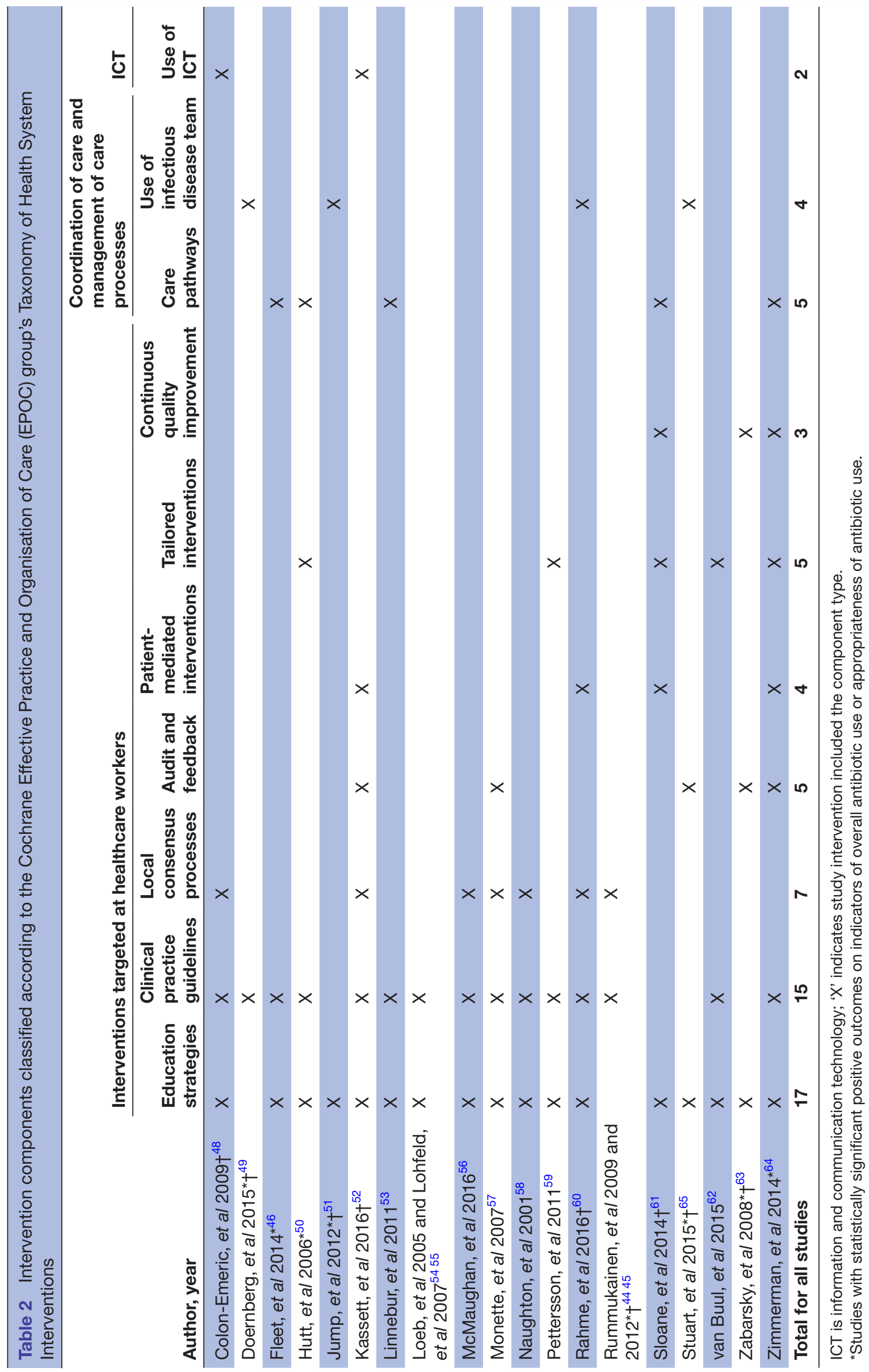




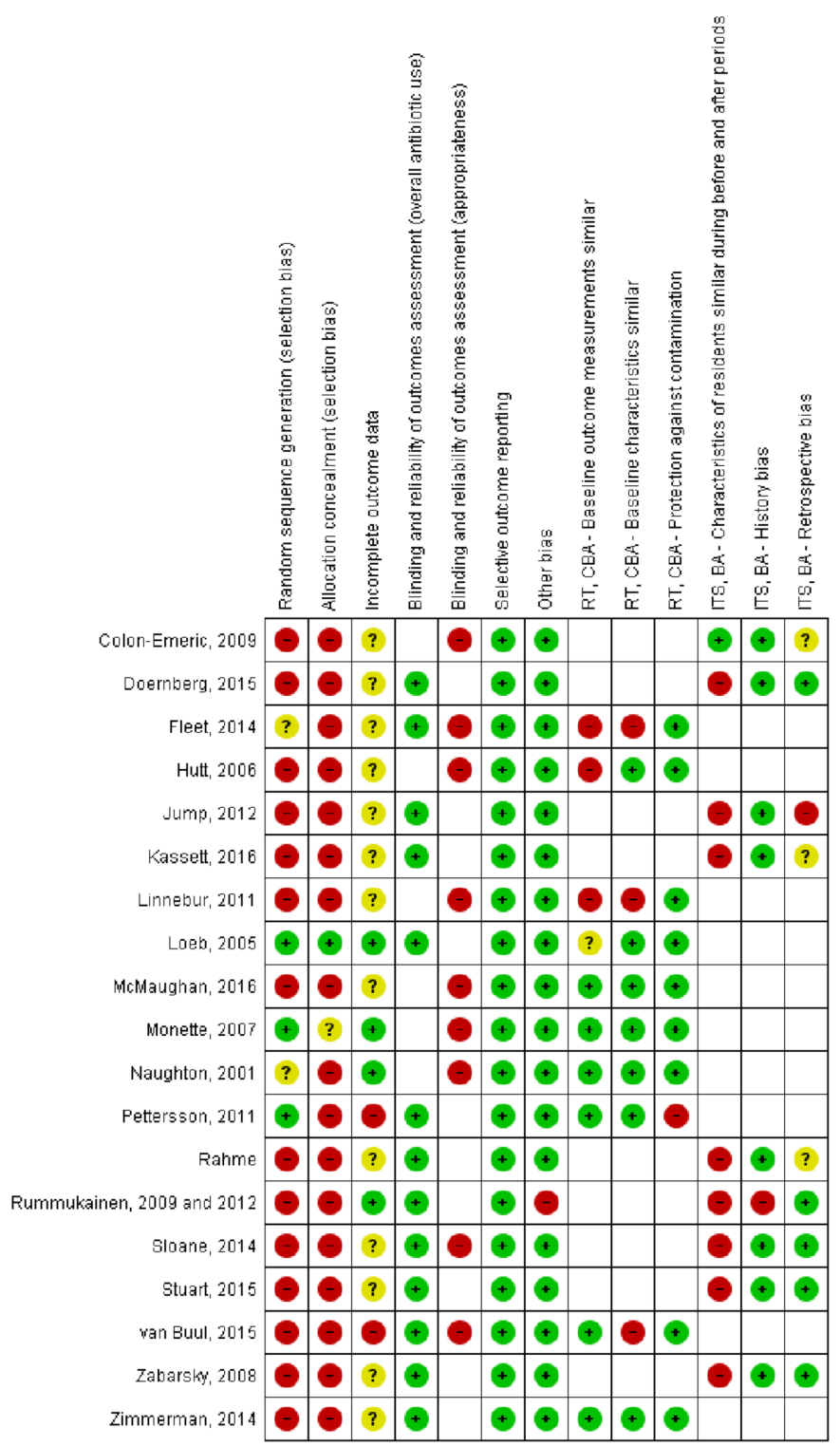

Figure 2 Risk of bias assessment results for each study. Blank sections in this graph are due to criteria not being applicable to all studies. RT, CBA - criterion applicable to randomised trials and controlled before-and-after studies. ITS, BA - criterion applicable to before-after and interrupted time series studies.

without controls and all eight studies were rated as having a high risk of bias (table 2).

\section{Effectiveness of interventions to reduce the overall antibiotic} use among LTCF residents

Twelve studies ${ }^{45} 49515459-65$ reported the impact of interventions on overall antibiotic use. Three ${ }^{454659}$ of these reported changes in the percentage of residents on an antibiotic, three 466062 changes in the DDD per 1000 resident days, three ${ }^{516365}$ changes in DOT per 1000 resident days and five ${ }^{454616264}$ changes in the number of antibiotic prescriptions per 1000 resident days.

Only the three studies ${ }^{45459}$ reporting changes in the percentage of residents on antibiotics after the intervention contained sufficient information to be included in a

\begin{tabular}{|c|c|c|c|c|c|c|}
\hline Author $(\mathbf{s})$ and Year & $\begin{array}{l}\text { e interve } \\
\text { Event }\end{array}$ & $\begin{array}{l}\text { intion } \\
\text { Total }\end{array}$ & $\begin{array}{l}\text { ter inter } \\
\text { Event }\end{array}$ & & & Relative Risk $995 \%$ Cl] \\
\hline Fleet et al, 2014 & 53 & 825 & 55 & 838 & - & $1.02[0.71,1.47]$ \\
\hline Rummukainen et al.,2012 & 203 & 1221 & 149 & 1211 & $=$ & $0.74[0.61,0.90]$ \\
\hline Pettersson et al., 2011 & 363 & 1394 & 315 & 1373 & $=$ & $0.88[0.77,1.00]$ \\
\hline \multirow{3}{*}{\multicolumn{5}{|c|}{ RE Model for All Studies $\left(Q=3.18, \mathrm{df}=2, \mathrm{p}=0.20 ; \mathrm{I}^{2}=34.2 \%\right)$}} & -1 & $0.85[0.61,1.18]$ \\
\hline & & & & & $0.3 \quad 12$ & \\
\hline & & & & & Relative Risk & \\
\hline
\end{tabular}

Figure 3 Forest plot of studies reporting intervention effect on the percentage of residents on antibiotics. Events are number of residents on antibiotics and total was number of residents. RE is random effects.

meta-analysis. All three studies were rated as having a high risk of bias. One ${ }^{59}$ of these studies showed a small reduction (RR: $0.74 ; 95 \%$ CI: 0.61 to 0.90 ) in residents' use of antibiotics (figure 3 ). The three studies were found to be homogeneous (heterogeneity between studies: $\mathrm{I}^{2}=34.2 \%$, $\mathrm{p}=0.2$ ). Overall, the interventions did not have a significant effect on residents' antibiotic use (pooled RR: 0.85, 95\% CI: 0.61 to $1.18, \mathrm{p}=0.2$ ) (figure 3 ). There was no evidence of publication bias $(\mathrm{p}=0.9)$. Sensitivity analyses were conducted using different post-intervention periods from Rummukainen et al (2012). ${ }^{45}$ There was little difference in the results (post 24 months: pooled RR: 0.85, 95\% CI: 0.60 to $1.19, \mathrm{p}=0.2$; post 36 months: pooled RR: $0.83,95 \%$ CI: 0.52 to $1.33, \mathrm{p}=0.2$ ).

One of the three studies measuring intervention effects on DDD per 1000 resident days reported a statistically significant $5 \%$ reduction in total antibiotic use postintervention in comparison to the control group. ${ }^{46}$ The other two studies did not report statistically significant changes in DDD per 1000 resident days. ${ }^{60} 62$

The three uncontrolled studies reporting the DOT per 1000 resident days ${ }^{516365}$ all reported statistically significant reductions in antibiotic use ranging from $20 \%$ to $35 \%$. Two studies reporting intervention effects on the number of prescriptions per 1000 resident days reported statistically significant decreases in the order of $5 \%{ }^{49}$ and $28 \%,{ }^{64}$ but three further studies showed no change. ${ }^{546162}$

Detailed results are shown in online supplementary file 6.

\section{Effect of interventions on appropriateness of the decision to} treat with an antibiotic

Five studies ${ }^{46} 561-63$ reported on the impact of interventions on the appropriateness of decisions to treat infections with an antibiotic. Three of these were on decisions to treat any infection, ${ }^{46} 6162$ three for UTIs ${ }^{566263}$ and one for RTI. ${ }^{62}$ To assess whether antibiotic use was appropriate, four studies ${ }^{46566263}$ looked at adherence to various guidelines, and one ${ }^{61}$ used a panel of experts to assess each antibiotic orders.

The three studies ${ }^{46} 6162$ reporting the appropriateness of the decision to treat any infection with antibiotics contained sufficient information to be included in a meta-analysis; none of these studies reported a significant effect of the intervention on the appropriateness of the 


\begin{tabular}{|c|c|c|c|c|c|c|}
\hline \multicolumn{7}{|l|}{ A } \\
\hline Author(s) and Year & \multicolumn{2}{|c|}{$\begin{array}{l}\text { Before intervention } \\
\text { Episodes Total }\end{array}$} & \multicolumn{2}{|c|}{$\begin{array}{l}\text { Affer intervention } \\
\text { Episodes Total }\end{array}$} & \multicolumn{2}{|r|}{ Relative Risk $[95 \% \mathrm{Cl}]$} \\
\hline Sloane et al., 2014 & 22 & 30 & 47 & 55 & $=$ & $1.17[0.92,1.48]$ \\
\hline Fleet 2014 & 16 & 139 & 26 & 135 & $=$ & $1.67[0.94,2.98]$ \\
\hline van Buul et al, 2015 & 278 & 339 & 233 & 295 & 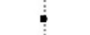 & $0.96[0.89,1.04]$ \\
\hline \multirow{2}{*}{\multicolumn{5}{|c|}{ RE Model for All Studies $\left(Q=5.42, \mathrm{df}=2, \mathrm{p}=0.07 ; \mathrm{P}^{2}=63.7 \%\right)$}} & $\frac{1}{\Gamma}$ & $1.10[0.64,1.91]$ \\
\hline & & & & & 0.6 & \\
\hline \multicolumn{7}{|c|}{ Relative Risk } \\
\hline
\end{tabular}

\begin{tabular}{|c|c|c|c|c|c|c|}
\hline \multicolumn{7}{|l|}{$B$} \\
\hline Author(s) and Year & $\begin{array}{r}\text { Before inten } \\
\text { Episodes }\end{array}$ & & $\begin{array}{c}\text { Atter interve } \\
\text { Episodes }\end{array}$ & $\begin{array}{l}\text { ention } \\
\text { Total }\end{array}$ & & Relative Risk $[95 \%$ Cl] \\
\hline Colon-Emeric, 2009 & 27 & 35 & 18 & 21 & - & $1.11[0.86,1.43]$ \\
\hline Hutt et al., 2006 & 13 & 21 & 19 & 24 & $=$ & $1.28[0.86,1.90]$ \\
\hline van Buul et al, 2015 & 10 & 110 & 8 & 81 & $=$ & $1.09[0.45,2.63]$ \\
\hline \multirow{3}{*}{\multicolumn{5}{|c|}{ RE Model for All Studies ( $Q=0.37$, df $=2, p=0.83 ; l^{2}=0.0 \%$ ) }} & - & $1.15[0.95,1.40]$ \\
\hline & & & & & 0.4113 & \\
\hline & & & & & \multicolumn{2}{|l|}{ Relattve Risk } \\
\hline
\end{tabular}

Figure 4 Forest plots of studies reporting intervention effect on the appropriateness of antibiotic use. Panel A: appropriateness of decision to treat any infection with antibiotics (episodes are number of episodes with appropriate treatment decision and total was number of orders). Panel B: appropriateness of antibiotic selection for respiratory tract infections (episodes are number of episodes on appropriate antibiotics and total was number of orders). $\mathrm{RE}$ is random effects.

decision to treat. All three studies were rated as having a high risk of bias. Overall, the intervention did not have a significant effect on the appropriateness of decisions to treat (pooled RR: $1.10,95 \%$ CI: 0.64 to $1.91, \mathrm{p}=0.5$ ) (figure 4 ). The studies were not found to have significant heterogeneity $(\mathrm{p}=0.07)$, but had a relatively high value of $\mathrm{I}^{2}(63.7 \%)$. There was evidence of publication bias $(\mathrm{p}=0.01)$. The sensitivity analysis showed little difference in the results when using the partial appropriateness assessment results by Fleet et al $(2014)^{46}$ (pooled RR: 0.98, 95\% CI: 0.78 to $1.24, \mathrm{p}=0.8$ ).

Three studies examined the impact of interventions on the decision to treat UTIs or asymptomatic bacteriuria. ${ }^{56} 6263$ Only one before-after study showed a statistically significant reduction in inappropriate decisions to treat asymptomatic bacteriuria with antibiotics (from $67.6 \%$ to $44.0 \%, \mathrm{p}=0.022$ ), while the two other studies showed no statistically significant improvement in the appropriateness of decisions to treat UTIs or asymptomatic bacteriuria with antibiotics. ${ }^{562}$

One study examined the proportion of RTIs appropriately treated with an antibiotic and reported no changes after the intervention compared with the control facilities (odds ratio: 0.97 ; $95 \%$ CI: 0.42 to 2.27 ) ${ }^{62}$ However, $89 \%$ of treatment decisions were deemed appropriate at baseline, leaving little room for improvement.

Detailed results are shown in online supplementary file 7.
Effect of interventions on appropriateness of the antibiotic selected

Six studies 485053576162 reported on the impact of interventions on the appropriateness of antibiotic selection. $\mathrm{Two}^{5761}$ of these reported on antibiotic selection for any infection, two ${ }^{48} 62$ for UTIs and four ${ }^{48505362}$ for RTIs. To assess whether antibiotic selection was appropriate, five studies $^{48} 50535762$ used various guidelines, and one ${ }^{61}$ an expert panel to assess antibiotic orders.

Only three of four studies ${ }^{485052}$ reporting the effects of interventions on the appropriateness of antibiotic selection for the treatment of RTI contained sufficient information to be included in this meta-analysis; none of these studies reported a significant effect on appropriateness of antibiotic selection (RTI). All three studies were rated as having a high risk of bias. The studies were found to be homogeneous (heterogeneity between studies: $\mathrm{I}^{2}=0 \%$, $\mathrm{p}=0.8$ ). Overall, the intervention did not have a significant effect on the appropriateness of antibiotic selection (pooled RR: 1.15, 95\% CI: 0.95 to 1.40, $\mathrm{p}=0.09$ ) (figure 4). There was no evidence of publication bias $(p=0.9)$.

The one study reporting the effect of the intervention on the appropriateness of antibiotic selection for RTI that was not included in the meta-analysis, also did not show a statistically significant improvement in this outcome. ${ }^{53}$

The two studies reporting intervention effects on the appropriateness of antibiotic selection for any indication showed no improvements post-intervention. ${ }^{5761}$ However, they both reported high baseline levels of appropriate antibiotic selection at $84 \%^{57}$ and $90 \%{ }^{61}$ of antibiotic orders. The two studies reporting intervention effects on the appropriateness of antibiotic selection to treat UTIs also did not demonstrate a significant intervention effect. $^{4862}$

Detailed results are presented in online supplementary file 7 .

\section{Staff perceptions of interventions and levels of intervention uptake as reported in process evaluations}

Seven studies conducted process evaluations of their intervention. ${ }^{4850566162}$ One study used a qualitative interview approach to ascertain the perspectives of LTCF staff on the lack of effect of the intervention on the appropriateness of antibiotic prescribing. ${ }^{62}$ The key issues identified included the high baseline level of appropriate prescribing which was difficult to further improve, a lack of motivation on the part of the physicians to improve prescribing and high physician turnover in the facilities. Another study reported that physicians had positive opinions of the implemented computer algorithms, however there was infrequent use of the algorithms to support decision-making. ${ }^{48}$ A third study ${ }^{54}{ }^{55}$ identified a lack of buy-in from nurses, difficulty in getting physicians to change practices and family expectations of antibiotics as barriers to compliance with the implemented clinical guideline. Continued use of the guidelines after the discontinuation of the study was hampered 
by difficulties in changing long-standing practices and ensuring adequate training of new staff. ${ }^{545}$

Four other studies assessed the uptake of the interventions either by facilities or individuals targeted by the interventions. ${ }^{46505661}$ One of these studies implemented two forms to be completed by nursing staff for all residents newly commenced on an antibiotic. ${ }^{46}$ They found that on average the two forms were $100 \%$ complete in only $31 \%$ and $46 \%$ cases. Another study also examined the use of implemented forms by staff to communicate acute medical issues including infections. ${ }^{61}$ They found that uptake was low immediately following implementation of the intervention (less than half of all infections had a completed form), but increased over the 15 month study period to double the initial levels. Another study examined intervention component uptake by facilities and found that not all intervention components were implemented in the intervention facilities and that some components were implemented in the control group (eg, immunisation policy, emergency medication kit containing correct antibiotics).$^{50}$ Finally, McMaughan et $a l$ assessed the use of a decision-making aid for suspected UTIs implemented as part of their intervention. ${ }^{56}$ They found large differences between facilities in terms of the rates of use, with some facilities rarely using the aid (ie, in less than $25 \%$ of UTI cases). Their post-hoc analysis stratified facilities into high and low intervention adherence groups and showed a significant reduction in inappropriate antibiotic prescriptions among the high intervention adherence facilities, which was in contrast to their intention-to-treat analysis which showed no intervention effect.

\section{DISCUSSION}

\section{Main findings}

This systematic review and meta-analysis showed there is insufficient evidence supporting the effectiveness of interventions to reduce overall antibiotic use and increase the appropriateness of antibiotic use in LTCFs. Meta-analysis showed no statistically significant reductions in overall antibiotic use as measured by the percentage of residents on antibiotics. Most studies showed no reductions in other measures of overall antibiotic use, including DDD per 1000 resident days and the number of prescriptions per 1000 resident days. The two studies ${ }^{46} 64$ reporting significant changes on these outcomes showed very marginal improvements. Three before-after studies with a high risk of bias showed significant decreases in antibiotic DOT per 1000 resident days. ${ }^{516365}$ Interventions had little effect on the appropriateness of decisions to treat or on antibiotic selection, with only one study with a high risk of bias showing statistically significant improvements in the decision to treat with antibiotics. ${ }^{63}$

\section{Implications for practice and policy}

LTCFs are a challenging setting for antibiotic interventions due to factors such as doctors being off-site, older populations being more susceptible to infection and having less specific symptoms of infection and other stakeholders' (eg, family, staff) preferences for treatment with antibiotics. ${ }^{66-68}$ These factors may explain some of the findings from the process evaluations which reported that adoption of interventions by facilities and staff within the facilities was low, and varied between facilities. Furthermore, contributing factors including high staff turnover and lack of physician motivation to change prescribing behaviours can be difficult to overcome and pose sustainability challenges for interventions. Interventions such as education and implementation of clinical guidelines are unlikely to overcome these challenges, particularly in the relatively short period of time it takes to run a trial. Thus, knowledge of the local culture and context are important factors to consider when selecting and planning interventions. Furthermore, an understanding of the specific antibiotic prescribing patterns and issues in LTCFs will also assist in guiding where intervention efforts should be directed. For example, some studies had higher rates of appropriateness of decisions to treat infections with antibiotics compared with appropriate antibiotic selection, ${ }^{5862}$ and others reported high baseline rates of appropriate antibiotic prescribing, leaving little room for improvement. The variability in antibiotic prescribing among LTCF physicians has also been highlighted in the literature ${ }^{69}$ It would be useful for LTCFs to understand antibiotic use patterns prior to implementing interventions so that problem areas are targeted.

The interventions implemented in the included studies had varying components, targeted different stakeholders in LTCF resident's care and different infection types. This makes it difficult to ascertain which intervention component or combination of components are most likely to be effective. However, the studies which showed significant improvements in antibiotic prescribing had some common intervention components. These included audit and feedback, introduction of care pathways and an infectious diseases team being available to advise on prescribing. Despite this, it is not possible to draw any concrete conclusions about these intervention components since the studies in question were mostly uncontrolled studies which limits the ability to make causality links between the intervention and effects. Future studies should explore these components further to ascertain their effectiveness.

Since physicians involved in prescribing antibiotics in LTCFs are based in primary care practices, we can look toward some of the recent innovative approaches to improving antibiotic use in primary care. Recent studies have shown that periodic prescriber feedback with peer comparison is more effective in changing prescribing practices than other interventions, including education strategies. ${ }^{70-72}$ Peer comparison, which was not used in any of the studies included in this review, is a behavioural economics theory driven intervention that could be explored further in the LTCF setting. These interventions involve providing physicians who are high prescribers of 
antibiotics, feedback on their antibiotic prescribing levels relative to their peers. Such interventions require timely information on prescribing levels. ICT provides a good opportunity to obtain timely data, as well as a platform for providing feedback on antibiotic use and prescribing levels; however it is currently underexploited for these purposes in LTCFs.

\section{Strengths and limitations}

This review has several strengths. We conducted metaanalyses on the effects of antibiotic use interventions in LTCFs on measures of overall and appropriateness of antibiotic use. Furthermore, our results are grouped into key indicators which allows a clear summary of intervention outcomes. Our inclusion of the results of the process evaluations is unique, shedding some light on the challenges faced by LTCFs implementing antibiotic interventions, and aids in explaining some of the results. Another strength was our use of a broad search strategy to maximise capturing relevant studies.

The main limitation of this systematic review and meta-analysis is the small number of included studies. This is further amplified by the lack of consistency in the outcomes and descriptive statistics reported. Additionally, because of the lack of controlled studies, our meta-analyses included only results from the intervention groups of studies. The inclusion of studies other than randomised trials or controlled studies, while allowing us to present more results, also meant that the meta-analyses need to be interpreted keeping the risk of bias in mind. Furthermore, the methods for assessing the appropriateness of antibiotics varied between studies limiting the comparability and generalisability of these results.

Lastly, since we ran our searches in November 2018, we have identified three more articles describing beforeafter studies of interventions to improve antibiotic prescribing in LTCFs that meet our inclusion criteria. One study evaluated the impact of an educational intervention to improve antibiotic use for UTIs in 10 Canadian LTCFs and reported a 26\% decrease (adjusted incidence rate ratio $=0.74,95 \% \mathrm{CI}$ : 0.47 to 0.74 ) in the overall number of antibiotic prescriptions per 1000 resident days. ${ }^{10}$ The second study, in seven Canadian LTCFs, reported a decrease in the use of antibiotics for asymptomatic bacteriuria from $90 \%$ pre-intervention to $62.9 \%$ post-intervention $(\mathrm{p}=0.003)$, following an educational intervention. ${ }^{73}$ The third study reported that the implementation of new guidelines and physician-pharmacynurse quality circles resulted in a $22 \%$ reduction in overall antibiotic use over a 6-year period. ${ }^{74}$ However, it is not possible to attribute the reductions in antibiotic use to the interventions alone due to the absence of a control group, and the inclusion of these studies in our review would not change our conclusions.

\section{Future research}

High quality studies are required to test the effectiveness of interventions to improve antibiotic use in LTCFs. Study designs should include controls as otherwise it is very difficult to account for external factors influencing outcomes in health services research. This fact is driven home by two of the included studies which showed improvements in outcomes in the control LTCFs, as well as the intervention LTCFs. ${ }^{59} 62$ Without comparison to the control groups, drawing conclusions about the effects of the interventions would be different. Planning of outcome measures also requires careful consideration. For example, indicators of overall antibiotic use measure different aspects of use $^{29} 7576$ and should be carefully selected based on the intervention planned. An intervention may not reduce the prevalence of antibiotic use, but may reduce the days of treatment, also an important outcome. Lastly, planned interventions should pay close attention that the implementation processes are relevant to the local context to ensure adequate intervention adoption in order to effect change. ${ }^{77}$ Carefully planned process evaluations can also assist with illuminating where and under what conditions the interventions are successful or otherwise ${ }^{78}$ Realistic evaluation is one such approach which considers intervention context, mechanisms and outcome in tandem. ${ }^{79}$

\section{CONCLUSIONS}

In this systematic review and meta-analysis, we reported that interventions were no more effective than usual care in improving antibiotic use in LTCFs. In the context of the global challenge of antibiotic resistance and ageing populations, the lack of evidence on interventions to improve antibiotic use in LTCFs is a major gap. The paucity of high quality studies and the heterogeneity in reported measures of antimicrobial use calls for more rigorous study designs, utilising a control group and standardised measures of reporting antibiotic consumption. Process evaluations are an important component of future studies in order to inform further intervention development and implementation in these complex settings.

Acknowledgements We would like to thank Mary Simons, Jeremy Cullis and Jane van Balen, Macquarie University Clinical Librarians for assistance with searching of the medical literature for this review.

Contributors MR conceived the study, the design of which was refined with MB and JW. CG led the design of the search strategy with input from MR. MR and $\mathrm{CG}$ conducted the data extraction, and $\mathrm{MR}$ and $\mathrm{MB}$ the risk of bias assessments. LL interpreted the extracted data and conducted the meta-analysis. MR led the drafting of the manuscript, which was reviewed for intellectual content by all coauthors who approved the final version to be published.

Funding MR is supported by an Australian Government National Health and Medical Research Early Career Fellowship (APP1143941).

Competing interests None declared.

Patient consent for publication Not required.

Provenance and peer review Not commissioned; externally peer reviewed.

Data availability statement All data relevant to the study are included in the article or uploaded as supplementary information. All data used in the metaanalysis are presented in the Supplementary materials.

Open access This is an open access article distributed in accordance with the Creative Commons Attribution Non Commercial (CC BY-NC 4.0) license, which permits others to distribute, remix, adapt, build upon this work non-commercially, and license their derivative works on different terms, provided the original work is 
properly cited, appropriate credit is given, any changes made indicated, and the use is non-commercial. See: http://creativecommons.org/licenses/by-nc/4.0/.

\section{ORCID iDs}

Magdalena Z Raban http://orcid.org/0000-0002-6995-2849

Melissa T Baysari http://orcid.org/0000-0003-1645-9126

\section{REFERENCES}

1 Wellcome Trust \& UK Government. Tackling drug-resistant infections globally: final report and recommendations, 2016. Available: https:// amr-review.org/sites/default/files/160525_Final\%20paper_with\% 20cover.pdf

2 Antoñanzas F, Goossens $\mathrm{H}$. The economics of antibiotic resistance: a claim for personalised treatments. Eur J Health Econ 2019;20:483-5.

3 Arvanitis M, Anagnostou T, Kourkoumpetis TK, et al. The impact of antimicrobial resistance and aging in VAP outcomes: experience from a large tertiary care center. PLoS One 2014;9:e89984.

4 Founou RC, Founou LL, Essack SY. Clinical and economic impact of antibiotic resistance in developing countries: a systematic review and meta-analysis. PLoS One 2017;12:e0189621.

5 Patel G, Huprikar S, Factor SH, et al. Outcomes of carbapenemresistant Klebsiella pneumoniae infection and the impact of antimicrobial and adjunctive therapies. Infect Control Hosp Epidemiol 2008;29:1099-106.

6 Yamamoto M, Pop-Vicas AE. Treatment for infections with carbapenem-resistant Enterobacteriaceae: what options do we still have? Crit Care 2014;18:229.

7 Marra F, McCabe M, Sharma P, et al. Utilization of antibiotics in longterm care facilities in British Columbia, Canada. J Am Med Dir Assoc 2017;18:1098.e1-1098.e11.

8 Thornley T, Ashiru-Oredope D, Normington A, et al. Antibiotic prescribing for residents in long-term-care facilities across the UK. $J$ Antimicrob Chemother 2019;74:1447-51.

9 van Buul LW, van der Steen JT, Veenhuizen RB, et al. Antibiotic use and resistance in long term care facilities. J Am Med Dir Assoc 2012;13:568.e1-568.e13.

10 Brown KA, Chambers A, MacFarlane S, et al. Reducing unnecessary urine culturing and antibiotic overprescribing in long-term care: a before-and-after analysis. CMAJ Open 2019;7:E174-81.

11 Falcone M, Paul M, Yahav D, et al. Antimicrobial consumption and impact of antimicrobial stewardship programmes in long-term care facilities. Clin Microbiol Infect 2019;25:562-9.

12 Mayne S, Sundvall P-D, Gunnarsson R. Confusion strongly associated with antibiotic prescribing due to suspected urinary tract infections in nursing homes. J Am Geriatr Soc 2018;66:274-81.

13 Donskey CJ, Sunkesula VCK, Stone ND, et al. Transmission of Clostridium difficile from asymptomatically colonized or infected long-term care facility residents. Infect. Control Hosp. Epidemiol. 2018;39:909-16.

14 Giufrè M, Accogli M, Ricchizzi E, et al. Multidrug-Resistant infections in long-term care facilities: extended-spectrum $\beta$-lactamaseproducing Enterobacteriaceae and hypervirulent antibiotic resistant Clostridium difficile. Diagn Microbiol Infect Dis 2018;91:275-81.

15 Kahvecioglu D, Ramiah K, McMaughan D, et al. Multidrug-Resistant organism infections in US nursing homes: a national study of prevalence, onset, and transmission across care settings, October 1, 2010-December 31, 2011. Infect Control Hosp Epidemiol 2014;35:S48-55.

16 Lim CJ, Cheng AC, Kennon J, et al. Prevalence of multidrug-resistant organisms and risk factors for carriage in long-term care facilities: a nested case-control study. Journal of Antimicrobial Chemotherapy 2014;69:1972-80.

17 March A, Aschbacher R, Dhanji $\mathrm{H}$, et al. Colonization of residents and staff of a long-term-care facility and adjacent acute-care Hospital geriatric unit by multiresistant bacteria. Clinical Microbiology and Infection 2010;16:934-44.

18 O'Fallon E, Schreiber R, Kandel R, et al. Multidrug-Resistant gram-negative bacteria at a long-term care facility: assessment of residents, healthcare workers, and inanimate surfaces. Infect. Control Hosp. Epidemiol. 2009;30:1172-9.

19 Luk S, Ho AYM, Chan EHY, et al. High prevalence and frequent acquisition of Clostridium difficile ribotype 002 among nursing home residents in Hong Kong. Infect Control Hosp Epidemiol 2018;39:782-7.

20 Davey P, Marwick CA, Scott CL, et al. Interventions to improve antibiotic prescribing practices for hospital inpatients. Cochrane Database Syst Rev 2017;2:Cd003543.
21 Roque F, Herdeiro MT, Soares S, et al. Educational interventions to improve prescription and dispensing of antibiotics: a systematic review. BMC Public Health 2014;14:1276.

22 Tonkin-Crine SK, Tan PS, van Hecke O, et al. Clinician-targeted interventions to influence antibiotic prescribing behaviour for acute respiratory infections in primary care: an overview of systematic reviews. Cochrane Database Syst Rev 2017;9:Cd012252.

23 Feldstein D, Sloane PD, Feltner C. Antibiotic stewardship programs in nursing homes: a systematic review. J Am Med Dir Assoc 2018;19:110-6.

24 Fleming A, Browne J, Byrne S. The effect of interventions to reduce potentially inappropriate antibiotic prescribing in long-term care facilities: a systematic review of randomised controlled trials. Drugs Aging 2013;30:401-8.

25 Moher D, Liberati A, Tetzlaff J, et al. Preferred reporting items for systematic reviews and meta-analyses: the PRISMA statement. Ann Intern Med 2009;151:264-9.

26 Centre for Reviews and Dissemination - University of York. Prospero: International prospective register for systematic reviews. Available: https://www.crd.york.ac.uk/prospero/ [Accessed $28 \mathrm{Sep}$ 2018].

27 Cochrane Collaboration. Cochrane library. Available: https://www. cochranelibrary.com/ [Accessed 28 Sep 2018].

28 Veritas Health Innovation. Covidence. Melbourne, Australia.

29 Centers for Disease Control and Prevention,. The core elements of antibiotic stewardship for nursing homes. US department of health and human services, 2015. Available: https://www.cdc.gov/ longtermcare/pdfs/core-elements-antibiotic-stewardship.pdf

30 European Centre for Disease Control. European Centre for Disease Prevention and Control, Antimicrobial Consumption - Annual Epidemiological Report for 2017, 2018. Available: https://www.ecdc. europa.eu/sites/portal/files/documents/AER_for_2017-antimicrobialconsumption.pdf

31 Public Health Agency of Canada. Public Health Agency of Canada, Canadian Antimicrobial Resistance Surveillance System - Update 2018, 2019. Available: https://www.canada.ca/content/dam/phacaspc/documents/services/publications/drugs-health-products/ canadian-antimicrobial-resistance-surveillance-system-2018-reportexecutive-summary/pub1-eng.pdf

32 Eriksen HM, Iversen BG, Aavitsland P. Prevalence of nosocomial infections and use of antibiotics in long-term care facilities in Norway, 2002 and 2003. $J$ Hosp Infect 2004;57:316-20.

33 Stevenson KB, Moore J, Colwell H, et al. Standardized infection surveillance in long-term care: interfacility comparisons from a regional cohort of facilities. Infect Control Hosp Epidemiol 2005;26:231-8

34 Cochrane Effective Practice and Organisation of Care Group,. The EPOC taxonomy of health systems interventions, in EPOC resources for review authors. Oslo: Norwegian Knowledge Centre for the Health Services, 2016

35 Cochrane Effective Practice and Organisation of Care Group. Suggested risk of bias criteria for EPOC reviews EPOC resources for review authors, 2017. Available: http://epoc.cochrane.org/resources/ epoc-resource-review-authors [Accessed 14 Sep 2018].

36 Casaroto E, Marra AR, Camargo TZS, et al. Agreement on the prescription of antimicrobial drugs. BMC Infect Dis 2015;15:248.

37 Mol PGM, Gans ROB, Panday PVN, et al. Reliability of assessment of adherence to an antimicrobial treatment guideline. $J$ Hosp Infect 2005:60:321-8

38 Sikkens JJ, van Agtmael MA, Peters EJG, et al. Assessment of appropriate antimicrobial prescribing: do experts agree? $J$ Antimicrob Chemother 2016;71:2980-7.

$39 \mathrm{Ho}$ AMH, Phelan R, Mizubuti GB, et al. Bias in before-after studies: narrative overview for Anesthesiologists. Anesth Analg 2018;126:1755-62.

40 Nordic Cochrane Centre. Review manager 5 (RevMan 5. Copenhagen: The Cochrane Collaboration, 2014.

41 Knapp G, Hartung J. Improved tests for a random effects metaregression with a single covariate. Stat Med 2003;22:2693-710.

42 Higgins JPTet al. Measuring inconsistency in meta-analyses. BMJ 2003;327:557-60.

43 Egger M, Smith GD, Schneider M, et al. Bias in meta-analysis detected by a simple, graphical test. BMJ 1997;315:629-34.

44 Rummukainen M, Jakobsson A, Karppi P, et al. Promoting hand hygiene and prudent use of antimicrobials in long-term care facilities. Am J Infect Control 2009;37:168-71.

45 Rummukainen $M-L$, Jakobsson $A$, Matsinen $M$, et al. Reduction in inappropriate prevention of urinary tract infections in long-term care facilities. Am J Infect Control 2012;40:711-4.

46 Fleet E, Gopal Rao G, Patel B, et al. Impact of implementation of a novel antimicrobial stewardship tool on antibiotic use in nursing 
homes: a prospective cluster randomized control pilot study. $J$ Antimicrob Chemother 2014;69:2265-73.

47 R Development Core Team,. cartographer R: a language and environment for statistical computing. Vienna, Austria: R Foundation for Statistical Computing, 2013.

48 Colon-Emeric CS, Schmader KE, Twersky J, et al. Development and pilot testing of computerized order entry algorithms for geriatric problems in nursing homes. J Am Geriatr Soc 2009;57:1644-53.

49 Doernberg SB, Dudas V, Trivedi KK. Implementation of an antimicrobial stewardship program targeting residents with urinary tract infections in three community long-term care facilities: a quasiexperimental study using time-series analysis. Antimicrob Resist Infect Control 2015;4:54.

50 Hutt E, Ruscin JM, Corbett K, et al. A multifaceted intervention to implement guidelines improved treatment of nursing Homeâ€"Acquired pneumonia in a state veterans home. J Am Geriatr Soc 2006;54:1694-700.

51 Jump RLP, Olds DM, Seifi N, et al. Effective antimicrobial stewardship in a long-term care facility through an infectious disease consultation service: keeping a lid on antibiotic use. Infect. Control Hosp. Epidemiol. 2012;33:1185-92.

52 Kassett N, Sham R, Aleong R, et al. Impact of antimicrobial stewardship on physician practice in a geriatric facility. Can $\mathrm{J}$ Hosp Pharm 2016;69:460-5.

53 Linnebur SA, Fish DN, Ruscin JM, et al. Impact of a multidisciplinary intervention on antibiotic use for nursing Home-Acquired pneumonia. Am J Geriatr Pharmacother 2011;9:442-50.

54 Loeb M, Brazil K, Lohfeld L, et al. Effect of a multifaceted intervention on number of antimicrobial prescriptions for suspected urinary tract infections in residents of nursing homes: cluster randomised controlled trial. BMJ 2005:331:669-72.

55 Lohfeld L, Loeb M, Brazil K. Evidence-Based clinical pathways to manage urinary tract infections in long-term care facilities: a qualitative case study describing administrator and nursing staff views. J Am Med Dir Assoc 2007;8:477-84.

56 McMaughan DK, Nwaiwu O, Zhao H, et al. Impact of a decisionmaking aid for suspected urinary tract infections on antibiotic overuse in nursing homes. BMC Geriatr 2016;16:1-9.

57 Monette J, Miller MA, Monette M, et al. Effect of an educational intervention on optimizing antibiotic prescribing in long-term care facilities. J Am Geriatr Soc 2007;55:1231-5.

58 Naughton BJ, Mylotte JM, Ramadan F, et al. Antibiotic use, hospital admissions, and mortality before and after implementing guidelines for nursing Homeâ€"Acquired pneumonia. J Am Geriatr Soc 2001:49:1020-4.

59 Pettersson E, Vernby A, Mölstad S, et al. Can a multifaceted educational intervention targeting both nurses and physicians change the prescribing of antibiotics to nursing home residents? a cluster randomized controlled trial. J Antimicrob Chemother 2011;66:2659-66.

60 Rahme CL, Jacoby HM, Avery LM. Impact of a hospital's antibiotic stewardship team on fluoroquinolone use at a long-term care facility. Annals of Long Term Care 2016;24:13-20.

61 Sloane PD, Zimmerman S, Reed D, et al. Antibiotic prescribing in 4 assisted-living communities: incidence and potential for improvement. Infect Control Hosp Epidemiol 2014;35:S62-8.

62 van Buul LW, van der Steen JT, Achterberg WP, et al. Effect of tailored antibiotic stewardship programmes on the appropriateness of antibiotic prescribing in nursing homes. $J$ Antimicrob Chemother 2015;70:2153-62.
63 Zabarsky TF, Sethi AK, Donskey CJ. Sustained reduction in inappropriate treatment of asymptomatic bacteriuria in a long-term care facility through an educational intervention. Am J Infect Control 2008;36:476-80.

64 Zimmerman S, Sloane PD, Bertrand R, et al. Successfully reducing antibiotic prescribing in nursing homes. J Am Geriatr Soc 2014;62:907-12.

65 Stuart RL, Orr E, Kotsanas D, et al. A nurse-led antimicrobial stewardship intervention in two residential aged care facilities. Healthc Infect 2015;20:4-6.

66 Lim CJ, Kwong MW-L, Stuart RL, et al. Antibiotic prescribing practice in residential aged care facilities--health care providers' perspectives. Med J Aust 2014;201:98-102.

67 Scales K, Zimmerman S, Reed D, et al. Nurse and medical provider perspectives on antibiotic stewardship in nursing homes. J Am Geriatr Soc 2017;65:165-71.

68 Schweizer AK, Hughes CM, Macauley DC, et al. Managing urinary tract infections in nursing homes: a qualitative assessment. Pharm World Sci 2005;27:159-65.

69 Daneman N, Campitelli MA, Giannakeas V, et al. Influences on the start, selection and duration of treatment with antibiotics in long-term care facilities. Can Med Assoc J 2017;189:E851-60.

70 Behavioural Economics Research Team - Government of Australia and Behavioural Economics Team of the Australian Government. Nudge vs superbug: a behavioural economics trial to reduce the Overprescribing of antibiotics. Commonwealth of Australia, 2018.

71 Gong CL, Hay JW, Meeker D, et al. Prescriber preferences for behavioural economics interventions to improve treatment of acute respiratory infections: a discrete choice experiment. BMJ Open 2016:6:e012739.

72 Hallsworth M, Chadborn T, Sallis A, et al. Provision of social norm feedback to high prescribers of antibiotics in general practice: a pragmatic national randomised controlled trial. The Lancet 2016;387:1743-52.

73 Lee C, Phillips C, Vanstone JR. Educational intervention to reduce treatment of asymptomatic bacteriuria in long-term care. BMJ Open Qual 2018;7:e000483.

74 Plüss-Suard C, Niquille A, Héquet D, et al. Decrease in antibacteria use and Facility-Level variability after the introduction of guidelines and implementation of Physician-Pharmacist-Nurse quality circles in Swiss long-term care facilities. J Am Med Dir Assoc 2019.

75 Monnet DL. Measuring antimicrobial use: the way forward. Clin Infect Dis 2007;44:671-3.

76 Polk RE, Fox C, Mahoney A, et al. Measurement of adult antibacterial drug use in 130 US hospitals: comparison of defined daily dose and days of therapy. Clin Infect Dis 2007;44:664-70.

77 Ford JH, Vranas L, Coughlin D, et al. Effect of a standard vs enhanced implementation strategy to improve antibiotic prescribing in nursing homes: a trial protocol of the improving management of urinary tract infections in nursing institutions through facilitated implementation (IMUNIFI) study. JAMA Netw Open 2019:2:e199526.

78 Moore GF, Audrey S, Barker M, et al. Process evaluation of complex interventions: medical Research Council guidance. $B M J$ 2015:350:h1258.

79 Salter KL, Kothari A. Using realist evaluation to open the black box of knowledge translation: a state-of-the-art review. Implementation Sci 2014;9:115. 